\title{
Kinematics of the Interstellar Vagabond 1I/‘Oumuamua (A/2017 U1)
}

\author{
Eric Mamajek ${ }^{1,2}$ \\ ${ }^{1}$ Jet Propulsion Laboratory, California Institute of Technology, M/S 321-100, 4800 Oak Grove Drive, Pasadena, CA 91109, USA \\ 2 Department of Physics 83 Astronomy, University of Rochester, Rochester, NY 14627, USA
}

Keywords: minor planets, asteroids: individual (1I/'Oumuamua) - stars: kinematics and dynamics 
The discovery of an asteroid of likely interstellar origin was recently made by the Pan-STARRS survey - A/2017 U1 $=1 \mathrm{I} /{ }^{\prime}$ Oumuamua ${ }^{1}$. Can 'Oumuamua's velocity before it entered the solar system provide any clues to its origin? The best available orbit from the JPL Small-Body Database Browser ${ }^{2}$ (solution JPL-13 produced by Davide Farnocchia) lists perihelion distance $q=0.255287 \pm 0.000079 \mathrm{au}$, eccentricity $e=1.19936 \pm 0.00021$ and semi-major axis $a=$ $-1.28052 \pm 0.00096 \mathrm{au}$. This value of $a$ is consistent with an initial velocity before encountering the solar system of $v_{\circ}=26.3209 \pm 0.0099 \mathrm{~km} \mathrm{~s}^{-1}$, assuming no non-gravitational forces. The ephemeris shows that the object entered the solar system from the direction $\alpha_{I C R S}, \delta_{I C R S}=279^{\circ} .804,+33^{\circ} .997\left( \pm 0^{\circ} .032, \pm 0^{\circ} .015 ; 1 \sigma\right)$. This divergent point and $v_{\circ}$ value translates to a heliocentric Galactic velocity (Perryman et al. 1998, $U$ towards Galactic center) of $U, V, W=$ $-11.457,-22.395,-7.746 \mathrm{~km} \mathrm{~s}^{-1}\left( \pm 0.009, \pm 0.009, \pm 0.011 \mathrm{~km} \mathrm{~s}^{-1}\right)$.

Could 'Oumuamua be a member of the Oort Cloud of the $\alpha$ Centauri system? Such a scenario might not be unexpected as the tidal radius for the $2.17 M_{\odot}^{N}$ triple system (Kervella et al. 2017) is of order $r_{t} \simeq 1.7$ pc (Mamajek et al. 2013). As the system lies only $1.34 \mathrm{pc}$ away, the solar system may be on the outskirts of $\alpha$ Cen's cometary cloud (see Hills 1981; Beech 2011). Kervella et al. (2017) calculated updated heliocentric Galactic velocities for $\alpha$ Cen AB of $U, V, W=-29.291,1.710,13.589( \pm 0.026, \pm 0.020, \pm 0.013) \mathrm{km} \mathrm{s}^{-1}$ and for Proxima Centauri ( $\alpha$ Cen C) of $U, V, W$ $=-29.390,1.883,13.777( \pm 0.027, \pm 0.018, \pm 0.009) \mathrm{km} \mathrm{s}^{-1}$. The velocity difference of $36.80 \pm 0.04 \mathrm{~km} \mathrm{~s}^{-1}$ between 'Oumuamua and the $\alpha$ Cen system, and the fact they were further apart in the past ( $\Delta \simeq 5$ pc 100 kyr ago), argues that it has no relation to $\alpha$ Cen. Members of $\alpha$ Cen's cometary cloud would appear to have motions diverging from the vicinity of $\alpha, \delta=293^{\circ},-42^{\circ}$ with $v_{\circ} \simeq 32 \mathrm{~km} \mathrm{~s}^{-1}$.

The Galactic velocity of 'Oumuamua is plotted against those of the nearest stars (parallax $>300$ mas) in Fig. 1. Besides the velocity of $\alpha$ Cen AB and C from Kervella et al. (2017), velocities for the nearest stars are drawn from Anderson \& Francis (2012) and Hawley et al. (1997). The velocity for the substellar binary Luhman 16 is calculated using data from Garcia et al. (2017) and Kniazev et al. (2013): $U, V, W=-18.3,-27.5,-6.9 \mathrm{~km} \mathrm{~s}^{-1}$. 'Oumuamua's velocity is more than $20 \mathrm{~km} \mathrm{~s}^{-1}$ from any of the stars, and $9 \mathrm{~km} \mathrm{~s}^{-1}$ off from Luhman 16, so 'Oumuamua does not appear to be comoving with any of these nearest systems.

What velocities might be expected of interstellar field objects? We might first suspect that interstellar planetesimals share the velocity distribution of nearby stars. The XHIP catalog (Anderson \& Francis 2012) contains velocities for 1481 stars within 25 pc with distances of $<10 \%$ accuracy. The XHIP sample has median velocity $U, V, W=-10.5$, $-18.0,-8.4 \mathrm{~km} \mathrm{~s}^{-1}\left( \pm 33, \pm 24, \pm 17 \mathrm{~km} \mathrm{~s}^{-1} ; 1 \sigma\right.$ range $)$, similar to that for volume-limited samples of nearby $\mathrm{M}$ dwarfs $\left(U, V, W=-9.7,-22.4,-8.9 \mathrm{~km} \mathrm{~s}^{-1} ; \pm 37.9, \pm 26.1, \pm 20.5 ; 1 \sigma ;\right.$ Reid et al. 2002). Bland-Hawthorn \& Gerhard (2016) provides a recent consensus estimate for the Local Standard of Rest (LSR) of $U, V, W=-10.0,-11.0,-7.0 \mathrm{~km} \mathrm{~s}^{-1} ; \pm 1$, $\pm 2, \pm 0.5 ; 1 \sigma)$. An object with the median velocity of the local XHIP sample would have speed $22.5 \mathrm{~km} \mathrm{~s}^{-1}$ coming from $\alpha, \delta=273^{\circ},+33^{\circ}$, within only $\sim 6^{\circ}$ of 'Oumuamua's divergent point. The velocity is very close to the median for the XHIP sample $\left(\Delta v \simeq 4.5 \mathrm{~km} \mathrm{~s}^{-1} ; \chi^{2} / \nu=0.036 / 3 ; \mathrm{P}=0.0018\right)$, the mean for the local M dwarfs $\left(\Delta v \simeq 2.1 \mathrm{~km} \mathrm{~s}^{-1}\right.$; $\left.\chi^{2} / \nu=0.0053 / 3 ; \mathrm{P}=0.0001\right)$ and the LSR $\left(\Delta v \simeq 11.5 \pm 2.3 \mathrm{~km} \mathrm{~s}^{-1}\right)$, compared to the typical 3D velocity of nearby stars. Compared to the LSR, 'Oumuamua has negligible radial and vertical Galactic motion ${ }^{3}$, and its sub-Keplerian circular velocity trails by $11 \mathrm{~km} \mathrm{~s}^{-1}$.

Robotic reconnaissance of 'Oumuamua, or future interstellar planetesimals passing through the solar system, might constitute logical precursors to interstellar missions, and provide the opportunity to conduct chemical studies and radiometric dating of extrasolar material which formed around other stars.

This research was carried out at the Jet Propulsion Laboratory, California Institute of Technology, under a contract with the National Aeronautics and Space Administration. EEM acknowledges support from the NASA NExSS pro-

\footnotetext{
${ }^{1}$ See: http://www.minorplanetcenter.net/mpec/K17/K17UI1.html, https://www.minorplanetcenter.net/mpec/K17/K17V17.html.

2 https://ssd.jpl.nasa.gov/sbdb.cgi?sstr=A\%2F2017\%20U1

${ }^{3}$ Gaidos et al. (2017) have proposed that the object's velocity is due to birth in a nearby 40 Myr stellar association.
} 

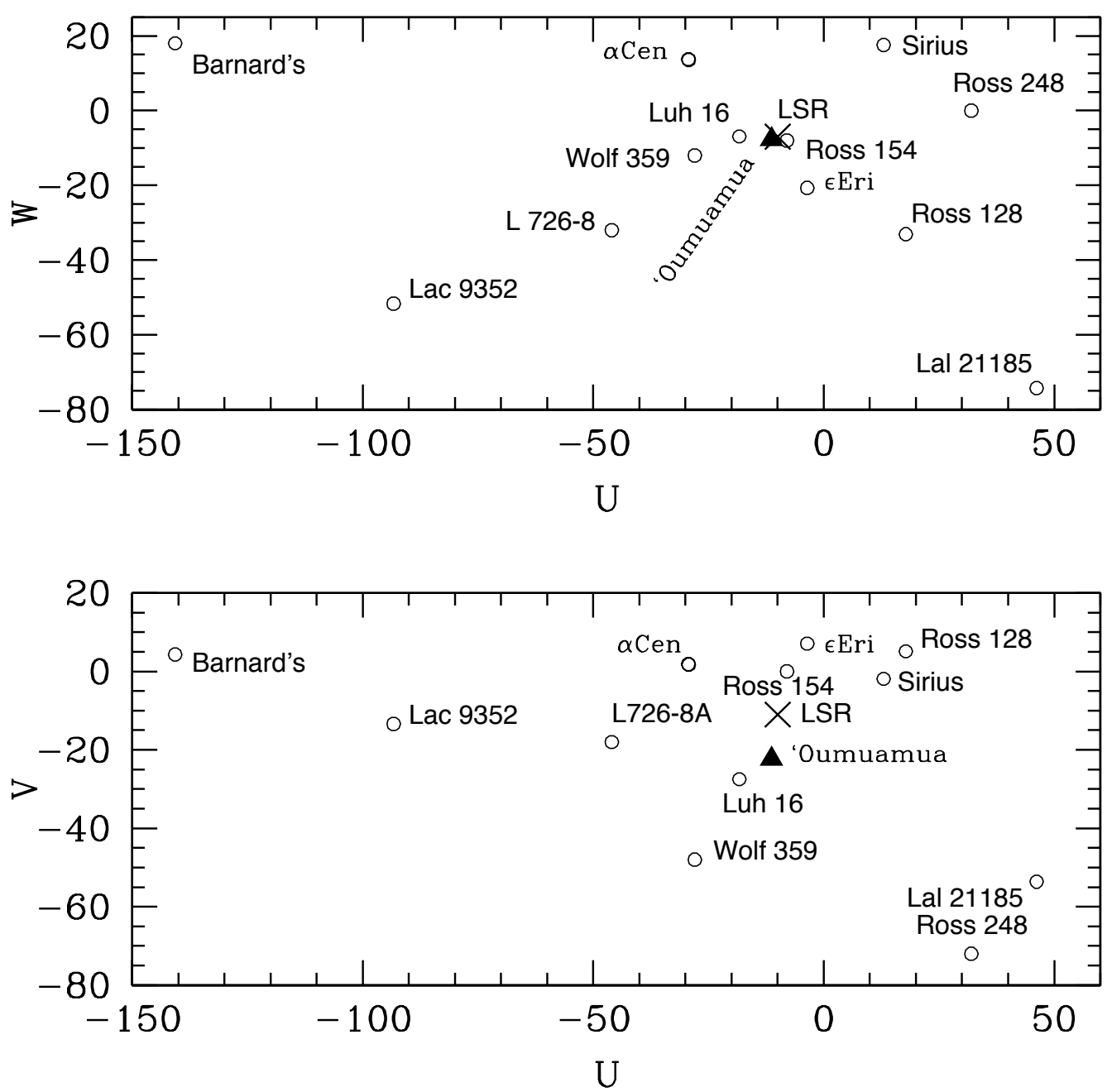

Figure 1. Galactic velocities for 1I/'Oumuamua (filled triangle), nearby stars (open circles), and LSR (cross).

gram, and thanks Davide Farnocchia (JPL) for discussions. This work used the JPL Small-Body Database Browser, HORIZONS system, and Vizier.

\section{REFERENCES}

Anderson, E., \& Francis, C. 2012, Astronomy Letters, 38, 331

Beech, M. 2011, The Observatory, 131, 212

Bland-Hawthorn, J., \& Gerhard, O. 2016, ARA\&A, 54, 529

Gaidos, E., Williams, J. P., \& Kraus, A. 2017, arXiv: 1711.01300

Garcia, E. V., Ammons, S. M., Salama, M., et al. 2017, ApJ, 846, 97

Hawley, S. L., Gizis, J. E., \& Reid, N. I. 1997, AJ, 113, 1458

Hills, J. G. 1981, AJ, 86, 1730
Kervella, P., Thévenin, F., \& Lovis, C. 2017, A\&A, 598, L7

Kniazev, A. Y., Vaisanen, P., Mužić, K., et al. 2013, ApJ, 770,124

Mamajek, E. E., Bartlett, J. L., Seifahrt, A., et al. 2013, AJ, 146, 154

Perryman, M. A. C., Brown, A. G. A., Lebreton, Y., et al. 1998, A\&A, 331, 81

Reid, I. N., Gizis, J. E., \& Hawley, S. L. 2002, AJ, 124, 2721 